\title{
La Nef des folles. Adaptation de Jean Drouyn et alii. Édition critique par Olga Anna Duhl
}

\section{Maria Colombo Timelli}

\section{(2) OpenEdition}

1 Journals

\section{Édition électronique}

URL : http://journals.openedition.org/studifrancesi/1096

DOI : 10.4000/studifrancesi.1096

ISSN : 2427-5856

Éditeur

Rosenberg \& Sellier

\section{Édition imprimée}

Date de publication : 1 novembre 2014

Pagination : $573-574$

ISSN : 0039-2944

\section{Référence électronique}

Maria Colombo Timelli, « La Nef des folles. Adaptation de Jean Drouyn et alii. Édition critique par Olga Anna Duhl », Studi Francesi [En ligne], 174 (LVIII | III) | 2014, mis en ligne le 01 novembre 2014, consulté le 17 septembre 2020. URL : http://journals.openedition.org/studifrancesi/1096 ; DOI : https://doi.org/ 10.4000/studifrancesi.1096

Ce document a été généré automatiquement le 17 septembre 2020.

\section{(c)}

Studi Francesi è distribuita con Licenza Creative Commons Attribuzione - Non commerciale - Non opere derivate 4.0 Internazionale. 


\title{
La Nef des folles. Adaptation de Jean Drouyn et alii. Édition critique par Olga Anna Duhl
}

\author{
Maria Colombo Timelli
}

\section{RÉFÉRENCE}

La Nef des folles. Adaptation de Jean Drouyn et alii. Édition critique par OLGA ANNA DUHL, Paris, Classiques Garnier, 2013 («Textes de la Renaissance» 190), pp. 390.

$1 \quad$ La Nef des folles est un traité en quelque sorte excentrique par rapport au riche corpus dérivé du Narrenschiff de Sebastian Brant (Bâle, 1494). Comme on le sait, ce traité connut un succès aussi rapide que vaste à l'échelle européenne: vite traduit en latin par Jacob Locher (Stultifera navis, Strasbourg, 1497) puis du latin en vers français par Pierre Rivière (Paris, 1497), cette dernière version fut encore adaptée en prose par Jean Drouyn (Lyon, 1498), alors que la version latine fut de nouveau traduite en prose française par un auteur resté anonyme (Paris, 1499-1500). Dans la foulée de cette fortune, Josse Bade acheva ses Stultiferae naves en 1498 déjà (editio princeps, Paris, 1501); à son tour, la traduction française de Jean Drouyn ne connut pas moins de six éditions entre 1500 et 1583, preuve d'une fortune durable au siècle de la Renaissance.

Ouvrage original à plusieurs égards, les Stultiferae naves - qui portent, comme le souligne O.A.D., «la double marque de l'humanisme italien et de la spiritualité de la devotio moderna» (p. 19) - sont fondées sur l'allégorie d'une flotte de cinq navires guidée par Ève, chaque navire représentant un sens personnifié par une femme folle. Après avoir rapidement introduit la tradition du texte (pp. 15-17), l'Introduction présente la biographie de Josse Bade (pp. 17-19), puis les traits originaux de son traité (pp. 19-26), ses «repères génériques» (pp. 27-29) et sa structure (pp. 30-36). Une deuxième partie est consacrée à la traduction française: problèmes de datation (pp. 36-38), auteur (pp. 38-39), «nouvelle orientation générique» (pp. 39-44), questions rattachées à une 
traduction qui se fait en même temps et inévitablement adaptation et remaniement (pp. 44-63). L'analyse linguistique (pp. 63-74) s'avère la section la plus faible et la plus confuse de cette partie: outre de nombreuses imprécisions, elle contient des naïvetés et des confusions dont il serait fastidieux de donner la liste. Je me borne à quelques exemples: dans le paragraphe consacré à la graphie, on lit "'Se' peut marquer le 'si' (concurrence des prononciations [se] et [si]» (p. 65); ou, à propos des adjectifs relatifsinterrogatifs: «'Dont' peut être utilisé comme pronom relatif» (p. 69); pour les pronoms démonstratifs, «la forme 'celluy' est concurrencée par la forme allongée [sic], issue du registre juridique, 'icelluy', utilisée souvent au sens adjectival»; et dans le même paragraphe: «'esquelz' apparait au sens de 'dans lesquels' [...], 'esquelles' veut dire 'en lesquelles' (sens adjectival)» (p. 69). L'Introduction comprend encore une analyse des bois gravés (pp. 74-75), sans que soit précisé quelle est l'édition examinée: il s'agit sans doute de celle de Paris, Petit Laurens pour Geoffroy de Marnef, ca 1498, qui fournit aussi le texte de base.

Un chapitre à part est consacré aux imprimés qui nous ont conservé La Nef des folles (pp. 77-83: six au total, dont cinq incunables); là encore, une certaine confusion règne, tout particulièrement entre «édition» et «exemplaire» (on lit, à propos de la princeps: «La reliure, française, est du XvIII ${ }^{e}$ siècle»; on imagine bien qu'il s'agit de l'exemplaire conservé dans la bibliothèque du Musée Condé, mais de fait il en existe au moins un deuxième à la Bodleienne d'Oxford: pp. 78-79). O.A. Duhl ajoute encore la liste des éditions connues des Stultiferae naves (pp. 83-85). Le choix du texte de base n'est pas très clair (cf. p. 86, «Choix des éditions» [sic, au pluriel]), l'éditrice affirmant: «Nous avons établi notre édition à partir de l'exemplaire de la bibliothèque du musée Condé $(A)$, celui de la BnF (B) et le texte de La Grand Nef des folz, traduction en prose de l'Anonyme de Marnef $(A M) » ;$ si $A$ correspond à la princeps, $B$ correspond apparemment à une édition différente (s.l. s.d., exemplaire BnF, Rés. M.Yc.750, numérisé dans Gallica, cf. p. 80), qui comprend, en plus, une «Exortation aux folles pour venir a la nef divine», éditée ici en appendice (pp. 259-262).

4 L'édition elle-même semble établie avec soin selon les principes énoncés p. 87; le texte est accompagné de notes explicatives et complémentaires en bas de page, alors que les variantes sont données à la fin (pp. 247-257). La Nef des folles est indubitablement un texte difficile, qui pose de nombreux problèmes d'édition - entre autres pour la ponctuation - et de compréhension, même pour un lecteur averti. Je propose quelques amendements aux pp. 101-105 de l'édition, qui contiennent «La declaration de la nef»; pour ce qui concerne la transcription, l'intégration de $r$ dans «plusieu[r]s» (p. 101) ne me parait pas indispensable; «on veoit plusieurs personnes qu'ilz mauldissent l'heure de leur naissance» (lire quilz, p. 103); la ponctuation devrait être modifiée p. 102: «nostre Seigneur [...] nous procrea nuds de toutes choses, ce qu'il n'a pas fait aux aultres creatures. Comme sont les bestes bruttes [...]. Car chescun quant et soy apporte son vestement»; deux virgules devraient remplacer les points. De même p. 103: «On voit que toutes bestes sentent leur nature et se gouvernent selon leur sentement. Car les unes sont farouches, cruelles, et maulvaises...»; je remplacerais le point par une virgule et supprimerais celle qui précède «et», reflet sans doute du système de ponctuation américain.

5 Une lecture des toutes dernières pages («L'excusation de l'acteur aux auditeurs», pp. 244-245) soulève le problème de l'utilisation des variantes; par exemple, si la leçon «parolle venir locuplete» paraît suspecte, c'est que la leçon correcte se lit dans AM: 
«parolle vayne 1.» (p. 257); dans l'invocation finale, «[que] nous puissons tous parvenir au benoist de salut», la lacune - non signalée - peut facilement être remplie par $A M$ toujours: «au b. port» (p. 257).

6 Vu la difficulté du texte, on aurait souhaité un glossaire plus riche. Signalons aussi une curiosité: si le glossaire occupe les pp. 263-280, les «locutions» sont données à part (pp. 281-283); cette dernière liste contient tant des adverbes (adonc / adoncques) et des conjonctions (jaçoit que), que des locutions verbales (adjouster foy, ne lui chaille [sic]), des images (plus saffres que liepars), voire des proverbes (tel a beaux yeulx qui ne voit goutte, glosé par 'être aveugle, être dans son tort [au sens moral]'); mais on y chercherait en vain seconde table ('pénitence', p. 105).

7 L'édition comprend encore: une riche Bibliographie (sources primaires, sources secondaires, histoire du livre, dictionnaires), qui rend compte des vastes connaissances d'olga Anna Duhl et de sa longue fréquentation de ce genre de textes (pp. 287-298), et des Index (noms propres, auteurs, lieux: pp. 299-306).

Il serait ingrat de ne pas reconnaitre l'importance du travail d'O.A.D., mais on ne peut manquer de souligner une fois de plus que l'édition critique exige des compétences que la connaissance approfondie d'un texte ne fournit pas automatiquement. Le moyen français, en l'occurrence le français du XvI ${ }^{\mathrm{e}}$ siècle, demeure une langue souvent opaque, tant dans sa morphosyntaxe que dans son lexique, et la philologie suit des règles qu'un critique littéraire - même le plus fin - se doit de maitriser lorsqu'il décide de se faire éditeur. 